\title{
Leishmaniose viscérale chronique au cours d'une chimiothérapie pour ostéosarcome métastatique
}

\author{
A Marguglio ${ }^{*}, \mathrm{C}$ Hoyoux ${ }^{2}, \mathrm{MF}$ Dresse $^{2}, \mathrm{JM}_{\text {Chantraine }}{ }^{3}$, A Thiry ${ }^{4}, \mathrm{P}_{\text {Gillet }}{ }^{5}$ \\ IÉtudiant 4 doctorat, aniversite de liege. ${ }^{+}$service d'anatomopathologie. \\ ${ }^{5}$ service de chirurgie. CHU Sart.Tilman, 4000 Liège, ${ }^{2}$ service d'hémato-oncologie pédiarrique, \\ service de pédiatrie. CHR Citudelle, 4000 Liègre, Belgique
}

(Reçu le 13 février 1997 : accepté le 6 novembre 1997)

\begin{abstract}
Résumé
On observe trois types de leishmanioses: la leishmaniose cutanée. la leishmaniose muqueuse et la leishmaniose viscérale, cette dernière étant provoquée par Leishmania donovani.

Observation. - Un enfant de 11 ans, d'origine thaillandaisc, résidant en Belgique depuis 6 ans, est opéré d'un ostéosarcome vertébral, métastasé au niveau pulmonaire, ce qui conduit à une polychimiothérapie suivie d'une métastasectomie pulmonaire. Un épisode d'hyperthermie, sans cause précise. avec altération de l'état général lors d'une aplasie médullaire postchimiothérapique est traité par une antibiothérapie à large spectre puis par amphotéricine b. L'évolution est d'abord favorable puis. 7 mois plus tard, un diagnostic de leishmaniose viscérale est posé à la suite de la réapparition des mêmes symptômes. Les traitements classiques par dérivés de l'antimoine (Glucantime ${ }^{\text {(i) }}$ ), puis par amphotéricine liposomale (Ambisome ${ }^{(i)}$ ) se montrent inefficaces. Une usssociation amphotéricine liposomale et interféron gamma amende les symptômes sans éradiquer le parasite de l'organisme. Face à cette leishmaniose viscérale chronique, le patient reçoit un traitement de maintenance par amphotéricine liposomale.

Conclusion. - Le caractère réfractaire et récidivant de cette leishmaniose viscérale chronique pourrait être secondaire au déficit immunitaire inhérent à la polychimiothérapie reçue en raison d'un ostéosarcome métastatique, actuellement en première rémission complète. 101998 Elsevier. Paris.
\end{abstract}

leishmaniose viscérale / ostéosarcome / déficit immunitaire / glucantime / amphotéricine

Summary - Chronic visceral leishmaniasis during chemotherapy for metastatic osteosarcoma.

Leishmaniasis refers to a spectrum of diseases caused by Leishmania. Clinically, three types of leishmaniavis can be distinguished: the cutaneous. mucoms and visceral leishmaniasis, the latter being caused by Leishmania donovani.

Case report. - An $I /$-year-old Thai. living in Belgium for 6 years, had surgery for a ventebral osteosarcoma with pulmonan metastuses, followed by polychemotherapy, then puimonar: metastasectomy. During a post-chemotherapy bone marrow aplasia, febrile episode with a general condition impairment was noted and first treated by broad-spectrum antibiotherapy, then by amphotericin $B$, in the absence of any accurate etiology. The outcome first was favorable. Nevertheless, 7 months later, the visceral leishmaniasis diagnosis was mate because of the recurrence of the same symptoms. Classical treatments by antimony: derivatives (Giucantim ${ }^{(\mathbb{3})}$ ), then liposomal amphoteritin (Ambisome ${ }^{\circledR}$ ) proved to be inefficient. A liposomal amphotericin-gamma interferon association suppressed the symptoms without eradicating the parasitc. The patient was given a maintenanie therapy based on liposomal amphote ricin.

Conclusion. - The stubborn and recurring nature of ihis chronic visceral leismaniosis can be due w the immune deficit inherent in the polvchemotherapy performed in onder to treat the metastatic osteoscroma which currently is in firs full remission. (1) 1998 Elsevier. Paris.

leishmaniasis, visceral / Leishmania donovani / sarcoma, osteogenic / immumosuppression / antimony / amphotericin B

\footnotetext{
* Correspondance et tirés à parl: A Marguglio, 122, rue de Jupille, B4620 Fléron (Liège).
} 
Les leishmanioses constituent un éventail d'affections causées par les leishmanies. Cliniquement, on observe trois types de leishmanioses: la leishmaniose cutanée, la leishmaniose muqueuse et la leishmaniose viscérale ou maladie de Kala-Azar [1]. La leishmaniose viscérale, dont l'agent causal est le plus souvent Leishmania donovani, transmis par piqûre du phlébotome, insecte vecteur de la maladie, se répand principalement sous forme d'épidémie en Inde, au Moyen-Orient, en Asie Centrale, en Afrique et au Brésil.

Seule la leishmaniose cutanée, causée par Leishmania infantum, touche l'Europe et, plus particulièrement, le sud de la France où 20 personnes environ sont affectées chaque année.

\section{OBSERVATION}

Un garçon d'origine thaïlandaise est adopté à l'âge de 4 ans et vit en Belgique depuis 1989. En juillet 1993, un diagnostic d'ostéosarcome de la septième vertèbre dorsale est posé et conduit à une vertébrectomie de D7. Le bilan d'extension réalisé objective des métastases pulmonaires bilatérales. Une polychimiothérapie (protocole T10 de Rosen) est administrée, suivie d'une métastasectomie pulmonaire bilatérale en deux temps. Cette chirurgie est consolidée par trois cures d'ifosfamide. L'ensemble du traitement est terminé en octobre 1994 et l'enfant est actuellement en première rémission complète.

Ce traitement s'est déroulé sans incident, à l'exception d'un épisode d'hyperthermie avec atteinte de l'état général, en août 1994, lors d'une aplasie médullaire postchimiothérapeutique. À cette occasion, le garçon présente une splénomégalie importante et, biologiquement, outre la pancytopénie, on note une hypergammaglobulinémie majeure $(\mathrm{IgG}=9450 \mathrm{mg} / \mathrm{dL}, \mathrm{IgM}=944 \mathrm{mg} / \mathrm{dL})$. Les recherches exhaustives, bactériologiques, virologiques et fongiques, restent négatives. Une antibiothérapie à large spectre puis de l'amphotéricine B amendent les symptômes et normalisent la biologie.

En février 1995, soit 4 mois après l'arrêt de la chimiothérapie, un nouvel épisode fébrile avec adénopathies cervicales et hépatosplénomégalie survient. Un diagnostic de toxoplasmose est posé à la suite de l'identification de toxoplasmes sur une biopsie ganglionnaire, malgré la sérologie spécifique négative. Un traitement classique par pyriméthamine et sulfadiazine entraîne une amélioration clinique transitoire.

Trois mois plus tard, la réapparition de l'hépatosplénomégalie conduit à la réalisation d'une ponction-aspiration de moelle osseuse, qui établit le diagnostic de leishmaniose viscérale, grâce à l'identification directe des parasites; la sérologie anti-Leishmania se révèle positive $(\mathrm{IgG}=640)$. Un traitement par dérivé de l'antimoine (Glucantime ${ }^{\circledR}$ $20 \mathrm{mg} / \mathrm{kg} / \mathrm{j}$ IV, en 2 heures ), pendant 20 jours, n'améliore ni la clinique ni la biologie, et le médullogramme de contrôle reste positif; ensuite, un traitement par amphotéricine liposomale (Ambisome ${ }^{\circledR} 3 \mathrm{mg} / \mathrm{kg} / \mathrm{j}$, pendant 7 jours, plus une dose de rappel au $21^{\mathrm{e}}$ jour) entraîne une lente régression des symptômes (baisse de la température, amélioration de l'état général en quelques jours, correction du syndrome inflammatoire) et la disparition des parasites au niveau de la moelle.

Cependant, 1 mois après l'arrêt du traitement par Ambisome ${ }^{\circledR}$, l'enfant présente une récidive de la symptomatologie; une nouvelle biopsie ganglionnaire confirme la présence des leishmanies. Simultanément apparaissent des troubles neuropsychométriques, de type troubles de l'attention, et des difficultés scolaires. Le scanner cérébral est normal; l'IRM du système nerveux central met en évidence des zones de démyélinisation bilatérales, symétriques, paraventriculaires, et des centres semi-ovales. Le liquide céphalorachidien est normal, à l'exception d'une hyperprotéinorachie à $19 \mathrm{mg} / \mathrm{dL}$; les cultures du liquide céphalorachidien (Leishmania, Borrelia, virus) sont négatives. Une association thérapeutique par Ambisome ${ }^{(\mathbb{B})}(1,5 \mathrm{mg} / \mathrm{kg} / \mathrm{j}$, pendant 21 jours $)$ et interféron gamma ( $100 \mu \mathrm{g} / \mathrm{m}^{2} / \mathrm{j}$, pendant 21 jours) amende les symptômes généraux et s'accompagne d'une régression des adénopathies cervicales, sans améliorer les troubles intellectuels.

Une biopsie de contrôle de ganglions cervicaux réalisée 8 semaines après ce traitement montre la persistance locale du parasite.

Devant cette leishmaniose viscérale chronique, un traitement de maintenance bimensuel par Ambisome ${ }^{(B)}$ ( $4 \mathrm{mg} / \mathrm{kg} / \mathrm{dose}$ en intraveineuse) est instauré.

Après 6 mois de traitement, les symptômes ont disparu, l'hypergammaglobulinémie également, la biologie est normalisée; seule la sérologie spécifique Leishmania reste positive.

\section{COMMENTAIRES}

Le garçon a développé à plusieurs reprises les signes habituels de la leishmaniose viscérale ou maladie de Kala-Azar (hyperthermie, altération de l'état général et splénomégalie majeure). La fièvre, qualifiée de fièvre folle, peut être d'allure très variable, avec des alternances de pics à $40^{\circ}$ entrecoupés de périodes d'hypothermie. Le premier épisode clinique de leishmaniose viscérale est probablement survenu en août 1994, lors d'une phase d'aplasie médullaire postchimiothérapeutique. Le diagnostic étiologique n'a pas été posé à cette occasion, mais la symptomatologie a vraisemblablement régressé grâce au traitement empirique par amphotéricine B.

Dans notre observation, l'agent responsable de la leishmaniose viscérale a été identifié par plusieurs ponctions-aspirations de la moelle osseuse; une première biopsie ganglionnaire n'a pas permis une visua- 
lisation optimale de la morphologie de ces parasites intracellulaires qui ont été confondus, dans un premier temps, avec des toxoplasmes (diagnostic infirmé par la sérologie); une empreinte ganglionnaire sur lame aurait peut-être permis l'identification plus précoce de ces leishmanies.

Le diagnostic de leishmaniose viscérale a été confirmé chez notre patient par la sérologie. Cette sérologie Leishmania est relativement spécifique ; seules le paludisme et la maladie de Chagas peuvent s'accompagner de faux positifs; ces deux diagnostics sont exclus chez notre patient.

En cours d'évolution, le garçon a développé un tableau neurologique de type régression psychométrique et intellectuelle. La responsabilité spécifique des leishmanies dans cette symptomatologie ne peut ètre affirmée en raison de la négativation de la culture et de la sérologie du liquide céphalorachidien. À notre connaissance, les lésions du système nerveux central ne sont pas classiquement décrites dans l'évolution de la leishmaniose viscérale; néanmoins, il faut préciser qu'aucune étude ne fait mention d'exploration cérébrale par IRM. Le scanner du système nerveux central du garçon est normal, suggérant des lésions minimes, pouvant passer inaperçues en l'absence d'examen technique plus performant. L'IRM permet d'envisager des zones de démyélinisation de la substance blanche de l'encéphale compatibles avec une origine infectieuse.

Bien que la culture du liquide céphalorachidien n'ait pas révélé la présence du parasite, la responsabilité des leishmanies dans la genèse de ces lésions ne nous paraît pas exclue. Le parasite pourrait éventuellement pénétrer dans le système nerveux central selon les mêmes modalités que le virus VIH, par l'intermédiaire des cellules du système réticuloendothélial, contaminées par l'agent infectieux.

Le tropisme neurologique de la leishmaniose viscérale est connu. En effet, Hashim et al [2] ont décrit une neuropathie périphérique mixte; notons que dans cette étude, les biopsies nerveuses sont restées négatives. Soulignons par ailleurs qu'une amélioration des performances intellectuelles fut constatée chez ce garçon sous Ambisome ${ }^{\circledR}$.

La source de contamination du garçon par les leishmanies reste énigmatique. Ni la Belgique, ni la Thälande ne constituent un foyer endémique connu de leishmaniose viscérale; quelques cas sporadiques ont été décrits en Belgique depuis 1990. Depuis son arrivée en Belgique (1989), l'enfant n'a pas quitté le territoire. Une contamination lors de ses premières années vécues en Thaïlande aurait probablement entraîné l'apparition des premiers signes de l'affection plus précocement (la période d'incubation de la maladie est en moyenne de 6 semaines à 6 mois).
Néanmoins, certains cas de portages sains ont été décrits; ces infections inapparentes résultent d'une cohabitation asymptomatique entre le parasite et l'hôte. À l'occasion d'un fléchissement des défenses immunitaires (greffes d'organes, VIH, chimiothérapie...) et en l'absence de tout nouveau contact infectieux, ces patients peuvent développer la maladie.

Une recherche exhaustive a été réalisée chez le garçon et n'a mis en évidence aucune infection concomitante (VIH...); une exploration immunitaire complète s'est avérée normale, à l'exception d'un nombre diminué de cellules $T$ helper $\left(110 / \mathrm{mm}^{3}\right)$. Cette diminution du nombre de lymphocytes $\mathrm{T} 4$ pourrait être secondaire à la chimiothérapie. En outre, il est connu que la leishmaniose viscérale peut s'accompagner d'une diminution significative du nombre et des fonctions des cellules $T$. Par conséquent, le rôle de l'immunodépression attribuable à la néoplasie et à la chimiothérapie apparaît dès lors fort probable dans le déclenchement de la maladie. Depuis 6 mois, l'enfant est sous traitement bimensuel par Ambisome ${ }^{\circledR}$ et le nombre de T4 est actuellement normalisé.

Plusieurs molécules thérapeutiques (Fungizone $\left.{ }^{(}\right)$, Glucantime ${ }^{\circledR}$, Ambisome $\left.{ }^{\circledR}\right)$ ont été successivement administrées au garçon; elles ont conduit à une amé.lioration clinique transitoire, mais sans éradication définitive du parasite. La résistance primaire de la leishmaniose viscérale aux dérivés de l'antimoine (Glucantime ${ }^{\circledR}$ ) s'observe chez $16 \%$ des sujets (cela dans la plupart des régions épidémiques et particulièrement l'Inde et l'Afrique), voire $25 \%$ des malades en cas d'immunodéficience, notamment causée par le VIH. Certains patients réfractaires répondent favorablement à l'administration simultanée de l'agent antiinfectieux et de l'interféron gamma; ce dernier, dont la production par les lymphocytes semble déficiente chez les patients souffrant de leishmaniose viscérale, potentialiserait l'effet thérapeutique grâce à ces effets «activateurs » au niveau macrophagique [3].

Chez notre patient, l'effet bénéfique de cette association thérapeutique ne fut que transitoire. Devant le caractère récidivant de l'affection présentée par le garçon, celui-ci a été placé sous traitement de maintenance (dans notre cas par Ambisome ${ }^{\circledR}$ ), traitement (à vie, par amphotéricine B) préconisé par l'OMS chez les patients VIH souffrant de leishmaniose viscérale chronique ou récidivante [4-7]. Après 6 mois de traitement, les signes ont disparu, les paramètres biologiques sont normalisés; seule la sérologie Leishmania reste positive. Aucune étude actuelle ne nous permet de définir la durée précise du traitement d'entretien à envisager chez notre patient. Nous proposons de traiter le garçon pendant 1 an. La surveillance clinique et paraclinique nous permettra d'apprécier la rémission de la leishmaniose viscérale. 


\section{CONCLUSION}

Notre patient a une leishmaniose viscérale chronique stabilisée grâce à un traitement de maintenance par Ambisome ${ }^{\circledR}$. Le caractère réfractaire et récidivant de l'affection pourrait être secondaire au déficit immunitaire inhérent à la polychimiothérapie reçue en raison d'un ostéosarcome métastatique, actuellement en première rémission complète. La cause des troubles neuropsychologiques ainsi que des lésions de démyélinisation observées à l'IRM n'a pu être précisée; bien que la responsabilité de la leishmaniose ne soit pas formellement démontrée, celle-ci ne peut être exclue.

\section{RÉFÉRENCES}

1 Pearson DR, De Queiroy-Sousa A. Leishmania species. In: Mendel, ed. Visceral (Kala-Azar), cutaneous and mucosal Leishmania. New York: Livingstone Churchill ; 1995. p 2428-50
2 Hashim FA, Ahmed AE, El Hassan M et al. Neurologic changes in visceral leishmaniasis. Am J Trop Med Hyg 1995 ; 52 : 149-54

3 Lang T, Hellio R, Kaye PM, Antoine JC. Leishmania donovaniinfected macrophages: characterization of the parasitophorous vacuole and potential role of this organelle in antigen presentation. J Cell Sci $1994: 107: 2137-50$

4 Davidson RN, Di Marterio L. Liposomal amphotericin B (Ambisome) in Mediterranean visceral leishmaniasis: a multicenter trial. $Q J$ Med $1994 ; 87: 75-81$

5 Laguna F, Torre-Cisneros J, Moreno V, Villanueva JL, Valencia E. Efficacy of intermittent liposomal amphotericin $B$ in the treatment of visceral leishmaniasis, in patients infected with human immunodeficiency virus [letter]. Clin Infect Dis 1995 ; $21: 711-2$

6 Lazanas MC, Tsekes GA, Papandreou S et al. Liposomal amphotericin B for leishmaniasis treatment of AIDS patients unresponsive to antomonium compounds. AIDS $1993 ; 7$ : 1018-9

7 Lopez Dupla M, Gil-Aguado A, Lavilla-Uriol P et al. Efficacy of liposomal amphotericin B in the treatment and secondary prophylaxis of visceral leishmaniasis in HIV infected patients: report of two cases [letter]. J Antimicrob Chemother 1993; 32 : $657-9$

\section{Nouvelles brèves}

\section{La dysplasie cléidocrânienne est due à des mutations d'un gène codant pour un activateur transcriptionnel de la différenciation ostéo- blastique}

La dysplasie cléidocrânienne (DCC) est une affection transmise sur le mode autosomique dominant. Cliniquement, elle se caractérise par une absence ou une hypoplasie des clavicules, un retard de fermeture de la grande fontanelle associé à une brachycéphalie souvent marquée, des anomalies dentaires (retard d'éruption, dents surnuméraires), un retard de la maturation squelettique. Il s'agit, parmi les dysplasies squelettiques sans retentissement statural majeur, de l'une des plus fréquentes. Le gène en cause dans cette maladie vient d'être identifié $[1,2]$. Il y a 2 ans, deux équipes ont, presque en même temps, établi que le locus en cause est situé en $6 \mathrm{p} 21$. Parallèlement, un gène nommé CBFA1 (core binding factor-alpha 1) avait été également localisé en $6 \mathrm{p} 21$. La fonction de ce gène, homologue au gène runt de la drosophile (chez laquelle il intervient dans les processus de segmentation) a été élucidée très récemment : $C B F A l$ code pour un activateur transcriptionnel de la différenciation ostéoblastique baptisé OSF2/CBFA 1 [3]. Il était alors aisé d'imaginer que, par sa fonction et sa localisation, $G B F A l$ pouvait être le gène dont les mutations étaient à l'origine de la DCC. Deux enfants atteints de DCC ont été étudiés; leurs parents étaient indemnes. L'ADN génomique des deux enfants ayant été extrait, l'ensemble de la séquence codante du gène $C B F A l$ a été étudié. Une mutation faux sens a été identifiée chez chacun des enfants, alors qu'elle n'était pas trouvée, à l'échelle de l'ADN génomique, chez leurs parents. La localisation de ces deux mutations laissait penser qu'elles seraient susceptibles d'affecter la liaison de la protéine à l'ADN. Cette hypothèse a été vérifiée par des expériences in vitro. Ces résultats montrent que la DCC est due à un défaut de la différenciation ostéoblastique. Ils ont été confirmés par l'identification d'autres mutations du gène $C B F A 1$ chez des enfants atteints de DCC [1,2]. De plus, l'invalidation ciblée du gène cbfal provoque chez la souris, à l'état hétérozygote, un phénotype proche de celui de la DCC. Il apparaît ainsi que la DCC est vraisemblablement liée à un phénomène d'haplo-insuffisance au locus CBFA1, même si un effet dominant négatif ne peut être complètement exclu.

1 Lee B, Thirunavukkavasu K, Zhou L, Pastere L, Baldini A, Hecht J et al. Missense mutations abolishing DNA binding of the osteoblast - specific transcription factor OSF2/CBFAl in cleidocranial dysplasia. Nature Genet 1997; 16:307-10

2 Mundlos S, Otto F, Mundlos C, Mulliken JB, Aylsworth AS, Albright $S$ et al. Mutations involving the transcription factor CBFA1 cause cleidocranial dysplasia. Cell 1997; 89: 773-9

3 Ducy $P$, Zhang R, Geoffroy V, Ridall AL, Karsenty G. Osf $2 / \mathrm{cbfa} 1$ : a transcriptional activator of osteoblast differenciation. Cell $1997 ; 89: 747-54$

P Labrune Hôpital Antoine-Béclère, Clamart 